\title{
UV-B-induced photomorphogenesis in Arabidopsis
}

\author{
Jigang $\mathrm{Li}^{1,2}$, Li Yang ${ }^{1,2}$, Dan Jin ${ }^{2,3}$, Cynthia D. Nezames ${ }^{2}$, William Terzaghi ${ }^{2,4}$, Xing Wang Deng ${ }^{1,2 \bowtie ~}$ \\ ${ }^{1}$ Peking-Yale Joint Center for Plant Molecular Genetics and Agro-biotechnology, State Key Laboratory of Protein and Plant Gene \\ Research, College of Life Sciences, Peking University, Beijing 100871, China \\ ${ }^{2}$ Department of Molecular, Cellular, and Developmental Biology, Yale University, New Haven, CT 06520, USA \\ ${ }^{3}$ Key Laboratory of Biotechnology and Crop Quality Improvement of Ministry of Agriculture, Biotechnology Research Center, \\ Southwest University, Chongqing 400716, China \\ ${ }^{4}$ Department of Biology, Wilkes University, Wilkes-Barre, PA 18766, USA \\ $\triangle$ Correspondence: xingwang.deng@yale.edu \\ Received May 1, 2013 Accepted May 17, 2013
}

\begin{abstract}
Ultraviolet-B (UV-B) is a relatively minor component of sunlight, but can induce stress-related physiological processes or UV-B-specific photomorphogenic responses in plants. In the last decade, significant progress has been made in understanding the UV-B photomorphogenic pathway, including identification of the key components in the pathway, molecular characterization of UV-B photoreceptor and perception mechanism, and elucidation of the signal transduction mechanisms from the photoactivated UV-B receptor to downstream gene expression. This review summarizes the key players identified to date in the UV-B photomorphogenic pathway and their roles in mediating UV-B signal transduction.
\end{abstract}

KEYWORDS UV-B, photomorphogenesis, signal transduction, gene expression

\section{INTRODUCTION}

Sunlight is of utmost importance to plants, not only as the primary energy source for photosynthesis, but also as an environmental signal regulating their growth and development throughout their entire life cycle. Ultraviolet (UV) light is part of the electromagnetic spectrum, and can be divided into three different bands according to wavelength: UV-A (315-400 nm), UV-B (280-315 nm) and UV-C (<280 nm). Solar UV-C and most UV-B is absorbed by the stratospheric ozone layer, therefore only UV-A and some UV-B reaches the surface of the earth, and inevitably irradiates the sessile plants. Although UV-B is a relatively minor component of sunlight, which ac- counts for $<0.5 \%$ of total light energy reaching the earth's surface, it has the highest energy of the daylight spectrum and has a substantial impact on the biosphere (Jenkins, 2009; Heijde and UIm, 2012).

In general, UV-B can induce two distinct categories of responses in plants: stress responses and photomorphogenic responses. The type of responses that are induced by UV-B is primarily determined by the fluence rate of exposure, and also dependent on whether the plants have been acclimated by prior exposure to UV-B. High UV-B fluence rates induce stress-related physiological processes in plants, including DNA damage, production of reactive oxygen species (ROS), and impairment of cellular processes (Ulm and Nagy, 2005; Jenkins, 2009; Heijde and Ulm, 2012). These types of responses appear to be mediated by signaling pathways that are not specific to UV-B, because many of the responsive genes can be regulated by other stresses such as DNA damage, pathogen defense or wounding (Ulm and Nagy, 2005; Jenkins, 2009). By contrast, low UV-B fluence rates promote photomorphogenic responses in plants, characterized by the inhibition of hypocotyl growth, cotyledon expansion, biosynthesis of anthocyanins and flavonoids, and stomatal opening (Kim et al., 1998; Ulm and Nagy, 2005; Jenkins, 2009). These responses are mediated by a recently characterized photoreceptor UV RESISTANCE LOCUS 8 (UVR8), and are required for plants' acclimation to UV-B stress (Ulm and Nagy, 2005; Jenkins, 2009; Rizzini et al., 2011). It was shown recently that the two pathways are largely independent of each other, but both are important for plants to achieve full UV-B tolerance (Gonzalez Besteiro et al., 2011). However, it should be noted that there is substantial overlap in the fluence rates that initiate the different types of responses (Jenkins, 2009). 
The past decade has witnessed dramatic progress in understanding the mechanisms underlying UV-B-induced photomorphogenesis in higher plants. With the molecular characterization of UVR8 as a UV-B photoreceptor (Rizzini et al., 2011) and elucidation of its crystal structure (Christie et al., 2012; Wu et al., 2012), it has become increasingly clear how UV-B light signals are perceived and transduced to generate a wide range of photomorphogenic responses. This review aims to highlight some of the most recent progress in elucidating the molecular, cellular and biochemical mechanisms of UV-B perception and signal transduction in Arabidopsis.

\section{UVR8: A UV-B PHOTORECEPTOR}

The history of UVR8, a seven-bladed $\beta$-propeller protein, essentially parallels the history of molecular genetic analysis of the UV-B photomorphogenic pathway. UVR8 was firstly identified in a screen of the Arabidopsis mutants that were hypersensitive to UV-B light (Kliebenstein et al., 2002). Sequence analysis of the UVR8 protein revealed its homology to human guanine nucleotide exchange factor, Regulator of Chromatin Condensation 1 (RCC1). Subsequent studies identified more uvr8 alleles, and demonstrated that UVR8 is the first UV-Bspecific signaling component that is absolutely required for the UV-B photomorphogenic pathway (Brown et al., 2005; Favory et al., 2009). Transcriptome analysis revealed that UVR8 regulates a range of genes with important roles in UV protection and the repair of UV damage (Brown et al., 2005), thus explaining the sensitivity of uvr8 mutants to UV-B.

The UVR8 protein was shown to be localized in both the cytoplasm and nucleus, and UV-B irradiation promoted its accumulation in the nucleus (Brown et al., 2005; Kaiserli and Jenkins, 2007). Interestingly, the abundance of UVR8 seemed to be constitutive and not affected by any light treatments including UV-B, therefore UV-B irradiation may cause the redistribution of UVR8 in the cell (Kaiserli and Jenkins, 2007). Nuclear accumulation of UVR8 occurred very rapidly (within minutes) and at low fluence rates (Kaiserli and Jenkins, 2007). Nuclear localization was required for UVR8 activity, but constitutively nuclear-localized UVR8 with a nuclear localization signal (NLS) still required UV-B illumination to stimulate UVR8 function (Kaiserli and Jenkins, 2007). In the nucleus, UVR8 was shown to associate with the chromatin of UV-B-responsive genes, such as the promoter region of ELONGATED HYPOCOTYL 5 (HY5; see below), suggesting that UVR8 may be directly involved in the transcriptional regulation of its target genes (Brown et al., 2005; Cloix and Jenkins, 2008). UVR8 may associate with chromatin by interaction with histones, and it is notable that both UVR8 and human RCC1 share the feature of chromatin association (Brown et al., 2005; Cloix and Jenkins, 2008). However, the biological significance of UVR8 association with chromatin remains largely unknown and there is no evidence so far to support a direct role of UVR8 in transcriptional regulation.

Another important discovery regarding UVR8 activity upon
UV-B illumination was its interaction with the E3 ubiquitin ligase CONSTITUTIVELY PHOTOMORPHOGENIC 1 (COP1; see below). It was shown that UVR8 interacted directly with COP1 in the nucleus in a rapid, UV-B-dependent manner, and both proteins were required for the UV-B photomorphogenic response (Favory et al., 2009). Moreover, a 27 amino acid region near the C-terminus of UVR8 was shown to be both necessary and sufficient for interaction with COP1, and deletion of this region abolished UVR8 function in plants (Cloix et al., 2012). The importance of UVR8-COP1 physical interaction in UV-B signal transduction was further supported by the finding that single amino-acid changes in UVR8 and COP1 proteins that abrogate direct interaction with their partner proteins caused aberrant UV-B signaling (Favory et al., 2009).

The breakthrough of the field was made in 2011, when Rizzini et al. described that UVR8 was the long-sought-after UV-B photoreceptor. Before that, it had been discussed for many years whether plants possess such a UV-B-specific photoreceptor. The lack of information about its biochemical and photobiological properties and the fact that many cellular components (such as nucleic acids and proteins) absorb UV-B had hindered the identification of such a putative photoreceptor (Jenkins, 2009). Nevertheless, Rizzini et al. (2011) employed in vitro and yeast heterologous biochemical systems, as well as genetic approaches, to show that a UVR8 dimer is the UV-B receptor. The authors also proposed that a number of highly conserved tryptophan residues, with tryptophan285 as a key residue, serve as light sensors in UVR8. Upon illumination with UV-B, UVR8 rapidly becomes a monomer and interacts with COP1 (Rizzini et al., 2011). Therefore, UVR8 monomerization is the likely molecular mechanism of UV-B photo-activation, which leads to the interaction of UVR8 with COP1 and the relay of the UV-B signal.

The work by Rizzini et al. opened the door for rapid progress in this field. For example, the determination of the crystal structure of UVR8 enabled the elucidation of its photochemistry (Christie et al., 2012; Wu et al., 2012). It was shown that the homodimer of UVR8 is maintained by salt-bridge interactions between charged amino acids at the dimeric interaction surface. Unlike other known photoreceptors, two tryptophans, W233 and W285, collectively serve as the chromophore of UVR8, and are present at the dimeric interface adjacent to saltbridging amino acids. Absorption of UV-B photons by these two tryptophans destabilizes the salt-bridges, resulting in dimer dissociation and signal initiation (Christie et al., 2012; Wu et al., 2012). Similarly, the importance of these UVR8 chromophore tryptophans in vivo was recently demonstrated by a mutagenesis study, which showed that mutation of either tryptophan to alanine impaired photomorphogenic responses of UVR8 in transgenic Arabidopsis plants (O'Hara and Jenkins, 2012). Interestingly, the UVR8 photoreceptor is capable of redimerization in vivo, a process mediated by REPRESSOR OF UV-B PHOTOMORPHOGENESIS 1 (RUP1) and RUP2 (see below), thus restoring the homodimeric ground state (Heilmann and Jenkins, 2013; Heijde and UIm, 2013). 


\section{COP1: E3 UBIQUITIN LIGASE, A CRITICAL POSITIVE REGULATOR OF THE UV-B PHOTOMORPHOGENIC PATHWAY}

COP1, which encodes a conserved RING finger E3 ubiquitin ligase, was first cloned and characterized in the model plant Arabidopsis as a repressor of light-regulated plant development (Deng et al., 1991, 1992). After more than 20 years of study, COP1 has been shown to be involved in multiple processes in many different organisms, including plant development and mammalian cell survival, growth, and metabolism (Yi and Deng, 2005; Lau and Deng, 2012). COP1 contains three domains: a RING finger domain in its $\mathrm{N}$-terminal region, a WD40 repeat domain in its $\mathrm{C}$-terminus, and a coiled-coil domain in the middle (Deng et al., 1992; Yi and Deng, 2005). COP1 is a central repressor in light signaling which acts as an E3 ligase targeting several photomorphogenesis-promoting proteins for degradation, including HY5 (Osterlund et al., 2000) and HY5 HOMOLOG (HYH; Holm et al., 2002).

Despite the extensive studies of the roles of COP1 in photomorphogenesis induced by other wavelengths, the involvement of COP1 in UV-B-induced photomorphogenesis was first reported by Oravecz et al. in 2006. Interestingly, in contrast to its repressor function in photomorphogenesis induced by visible-light, COP1 acts as a critical positive regulator of UV-Binduced photomorphogenic responses (Oravecz et al., 2006). In addition, COP1 is required for the activation of $H Y 5$ gene expression in response to UV-B (Oravecz et al., 2006), whereas in dark-grown seedlings COP1 acts as an E3 ubiquitin ligase directly targeting $\mathrm{HY} 5$ for degradation (Osterlund et al., 2000). Moreover, COP1 accumulates in the nucleus in response to UV-B (Oravecz et al., 2006), also contrasting with the nuclear exclusion of COP1 in response to visible light (von Arnim and Deng, 1994; Subramanian et al., 2004). COP1 forms protein complexes with four WD40-repeat proteins, SUPPRESSOR OF PHYA-105 (SPA1) and its related proteins SPA2, SPA3, and SPA4 (Hoecker et al., 1998; Hoecker and Quail, 2001; Laubinger and Hoecker, 2003; Saijo et al., 2003; Seo et al., 2003; Laubinger et al., 2004; Zhu et al., 2008). In all other responses tested, the SPA proteins are required for COP1 function (Laubinger et al., 2004); however, the SPA proteins do not seem essential in the response to UV-B, and COP1 can function properly without the SPA proteins in the UV-B photomorphogenic pathway (Oravecz et al., 2006).

As mentioned above, both UVR8 and COP1 are required for UV-B-induced photomorphogenesis, and interaction of UVR8 monomers with COP1 may be the mechanism of UV-B signal transduction from photo-activated UVR8. However, how COP1 transduces the UV-B signal to downstream effectors remains largely unknown. There is no evidence so far that COP1 also acts as an E3 ubiquitin ligase in the UV-B photomorphogenic pathway. In addition, UVR8 seems to be quite stable (Kaiserli and Jenkins, 2007; Heilmann and Jenkins, 2013) and levels of UVR8 protein are similar in wild-type and cop1-4 mutant plants (Favory et al, 2009), suggesting that COP1 may not regulate the stability of UVR8 although it does so to other plant photoreceptors such as phytochromes and cryptochromes (Shalitin et al., 2002; Seo et al., 2004; Saijo et al., 2008; Jang et al., 2010).

\section{HY5: BZIP TRANSCRIPTION FACTOR, A PREDOMINANT DOWNSTREAM EFFECTOR OF THE UV-B PATHWAY}

HY5, a constitutively-nuclear bZIP protein, is the first known and most extensively studied transcription factor involved in promoting photomorphogenesis under a wide spectrum of wavelengths, including far-red (FR), red (R) and blue (B) light (Koornneef et al., 1980; Oyama, et al., 1997; Osterlund et al., 2000). It was shown that the abundance of HY5 protein is directly correlated with the extent of photomorphogenic development (Osterlund et al., 2000). Recent chromatin immunoprecipitation (ChIP)-chip studies revealed that HY5 directly binds to a large number of genomic sites, mainly at the promoter regions of annotated genes (Lee et al., 2007; Zhang et al., 2011). HY5 directly mediates both the up- and down-regulation of gene expression by light, and controls the expression of $\sim 20 \%$ of light-regulated genes in Arabidopsis (Ma et al., 2002). Therefore, HY5 is likely a fundamental hierarchical regulator of the transcriptional cascades involved in seedling photomorphogenesis (Lee et al., 2007).

The involvement of HY5 in UV-B-activated gene expression was first identified in a genome-wide expression analysis by UIm et al. in 2004. Subsequent studies established that HY5 is a key effector of the UV-B pathway since hy5 mutants displayed reduced tolerance to UV-B (Brown et al., 2005; Oravecz et al., 2006). A HY5p:LUC reporter (the firefly gene encoding luciferase driven by the HY5 promoter) was used to screen for mutants altered in UV-B-induced HY5 expression, and led to the identification of new cop1 and uvr8 alleles (Favory et al., 2009). This indicates that HY5 acts downstream of COP1 and UVR8, and is transcriptionally activated by UV-B in a UVR8- and COP1-dependent manner. It has been shown that $\mathrm{HY} 5$ and its homolog $\mathrm{HYH}$ redundantly regulated the expression of most UVR8-inducible genes, with HY5 playing a more predominant role (Brown et al., 2005; Brown and Jenkins, 2008). However, it was also shown that transcriptional activation of a subset of UVR8- and COP1-dependent genes was not dependent on HY5/HYH (Feher et al., 2011), indicating that other transcription factors may also be involved in transmitting UV-B signals. Other signaling intermediates of the UV-B photomorphogenic pathway are also emerging, such as SALT TOLERANCE (STO; also known as BBX24), a B-box zinc finger protein, which was shown to negatively regulate UV-B signaling by interacting with COP1 and repressing HY5's transcriptional activity (Jiang et al., 2009, 2012). In addition to transcriptional activation of $\mathrm{HY} 5$ expression, $\mathrm{HY} 5$ protein is also stabilized by UV-B, and it is evident that COP1-mediated degradation of HY5 is inhibited under UV-B (Favory et al., 2009). The underlying mechanisms remain to be investigated. 
It is not known whether UV-B-specific interaction of UVR8 with COP1 changes the E3 activity of COP1 or leads to reduced interaction of COP1 with HY5, which ultimately stabilizes HY5 protein in response to UV-B (Heijde and UIm, 2012).

\section{RUP1 AND RUP2: DWD-FAMILY PROTEINS, NEGATIVE REGULATORS OF THE UV-B PHOTOMORPHOGENIC PATHWAY}

RUP1 and RUP2 belong to the WD40-repeat protein superfamily, whose members contain at least one copy of a conserved motif (named WD40 motif) of $\sim 40$ amino acids typically ending in Trp-Asp (van Nocker and Ludwig, 2003). Within proteins the WD motif is typically found as multiple (4-10) tandemly repeated units. Both RUP1 and RUP2 consist of seven WD40-repeats without other apparent domains (Gruber et al., 2010). As mentioned above, the COP1 and SPA proteins also contain WD40-repeats, and together with RUP1 and RUP2 belong to a subfamily characterized by a conserved 16 -aa DDB1-binding WD40 (DWD) motif within the WD40 domain (Lee et al., 2008). Consistently, COP1 and SPA proteins were shown to interact with DDB1 and form a series of CUL4-DDB1COP1-SPA E3 ligase complexes (Chen et al., 2010). However, whether RUP1 and RUP2 could also interact and work in concert with DDB1 awaits further investigation.

Recently, it was shown that upon UV-B exposure the RUP1 and RUP2 genes were transcriptionally activated in a UVR8-, COP1-, and HY5-dependent manner (Gruber et al., 2010). The rup1 rup2 double mutants showed an enhanced response to UV-B and elevated UV-B tolerance, whereas overexpression of $R U P 2$ resulted in reduced UV-B responses and impaired acclimation (Gruber et al., 2010). Therefore, RUP1 and RUP2 act to repress the UV-B signaling pathway, and the direct interaction of RUP1 and RUP2 with UVR8 suggests that their repressive mechanism may be at the photoreceptor level (Gruber et al., 2010). Consistent with this assumption, a more recent study demonstrated that RUP1 and RUP2 mediate the redimerization of UVR8, which results in the disruption of the UVR8-COP1 interaction and reversion of the UVR8 monomers to the homodimeric ground state (Heijde and UIm, 2013). This UVR8 "off switch" mechanism facilitated by RUP1 and RUP2 could regenerate the reactivatable UVR8 homodimers, thus may play an important role for optimal growth and development of plants in sunlight (Heijde and Ulm, 2013; Heilmann and Jenkins, 2013). However, regarding whether or not COP1 is required for UVR8 redimerization, recent reports have reached different conclusions (Heilmann and Jenkins, 2013; Heijde and Ulm, 2013). Nevertheless, since the UV-B-responsive expression of RUP1 and RUP2 requires COP1 (Gruber et al., 2010), there is presumably sufficient basal level of RUPs if RUPSmediated UVR8 redimerization is independent of COP1.

\section{FHY3: TRANSPOSASE-DERIVED TRANSCRIPTION FACTOR, A NEW PLAYER IN UV-B SIGNALING}

FAR-RED ELONGATED HYPOCOTYL 3 (FHY3) and its homolog FAR-RED IMPAIRED RESPONSE 1 (FAR1) are transposase-derived transcription factors originally identified as components of phytochrome A (phyA) signaling (Whitelam et al., 1993; Hudson et al., 1999; Wang and Deng, 2002; Hiltbrunner et al., 2005, 2006; Lin et al., 2007; Genoud et al., 2008; Li et al., 2010). Studies conducted in recent years demonstrated that in addition to their critical role in phyA signaling, FHY3 and FAR1 also function redundantly in multiple developmental processes in plants, such as the circadian clock, chloroplast development, chlorophyll biosynthesis, and shoot branching, with FHY3 always playing a predominant role (Allen et al., 2006; Li et al., 2011; Ouyang et al., 2011; Stirnberg et al., 2012; Tang et al., 2012). Most of these processes are regulated by light, and the association of FHY3 with phyA in vivo (Saijo et al., 2008) suggests that FHY3/FAR1 may transmit the light signals directly from the photo-activated phytochromes. In addition, FHY3 and FAR1 were shown to work in concert with other transcription factors, such as HY5, PHYTOCHROME-INTERACTING FACTOR 1 (PIF1), CIRCADIAN CLOCK ASSOCIATED 1 (CCA1) and LATE ELONGATED HYPOCOTYL (LHY), to finetune the expression of their target genes (Li et al., 2010, 2011; Ouyang et al., 2011; Tang et al., 2012). The co-action of FHY3/ FAR1 with specific transcriptional repressors that regulate a distinct biological process may likely define a common molecular mechanism underlying FHY3/FAR1 action.

Recently, it was reported that COP1 is also inducible by UV-B (Huang et al., 2012). Interestingly, FHY3 and HY5 directly bind to their respective cis-elements present within the COP1 promoter, and play essential roles in UV-B induced expression of COP1 (Huang et al., 2012). The fhy 3 mutants displayed impaired UV-B-induced hypocotyl growth and reduced tolerance of UV-B damage, therefore FHY3 defines a new positive regulator in the UV-B signaling pathway. It was shown that both $\mathrm{FHY} 3$ and $\mathrm{HY} 5$ positively regulate the expression of COP1 (Huang et al., 2012), and because accumulation of HY5 in UV-B light requires COP1 (Favory et al., 2009), it is likely that HY5 promotes COP1 expression via a positive feedback loop. Unexpectedly, the far1 mutants showed no apparent impairment, and the fhy 3 far1 double mutants displayed no further impairment of UV-B-induced hypocotyl growth (Huang et al., 2012), indicating that FAR1 is not essential for the UV-B photomorphogenic pathway.

\section{UV-B-REGULATED GENE EXPRESSION}

In the last decade, transcriptome profiling analyses with maize (Casati and Walbot, 2003, 2004; Casati et al., 2006) and Arabidopsis (Brosche et al., 2002; Ulm et al., 2004; Brown et al., 2005; Kilian et al., 2007; Brown and Jenkins, 2008; Morales et al., 2013) revealed a large number of UV-B-responsive genes. Consistent with the two distinct types of responses that can be induced by UV-B, high fluence rates and short wavelengths of UV-B exposure induced many genes normally expressed in stress responses, including DNA damage, wounding and pathogen defense responses, whereas low fluence rates (includ- 
ing brief exposure) and long wavelengths of UV-B exposure stimulate a range of genes involved in UV protection or the amelioration of UV damage (UIm et al., 2004; Ulm and Nagy, 2005; Brown and Jenkins, 2008; Jenkins, 2009). These studies provide valuable insights into the understanding of the complex responses induced by UV-B, although the functions of most of these UV-B-regulated genes remain largely unclear. However, gene profiling studies followed by reverse genetic approaches facilitated the identification of new players in the UV-B photomorphogenic pathway, such as HY5 (Ulm et al., 2004). In addition, the identification of UV-B-responsive genes also made it possible to perform high-throughput genetic screens to isolate novel mutants that altered the UV-B-responsive gene expression, which was successfully used to identify new cop1 and uvr8 mutant alleles (Favory et al., 2009).

\section{PERSPECTIVE}

The last decade has seen significant progress in unraveling the key components and signaling mechanisms of the UV-B photomorphogenic pathway. The characterization of UVR8 as the UV-B photoreceptor has opened the door for a better understanding of the molecular, cellular and biochemical events in response to UV-B, from activation of the photoreceptor itself to downstream UV-B-responsive gene expression. However, several important questions still remain to be answered. For example, how is UVR8 nuclear accumulation controlled? It was shown that UVR8 nuclear accumulation could be observed within 5 min of UV-B exposure, and its N-terminal 23-aa region is required for efficient nuclear accumulation (Kaiserli and Jenkins, 2007). However, there is no NLS in this region. These observations invite the question of whether there is a nuclear transport system for UVR8, similar to the roles of FHY1 and FHY1-LIKE (FHL) in phyA nuclear accumulation (Hiltbrunner et al., 2005, 2006). In addition, the biological significance of UVR8 association with chromatin is not yet clear. Other photoreceptors, like phytochromes, have also been suggested to be associated with target gene promoters by interacting directly or indirectly with DNA-binding transcription factors (Martinez-Garcia et al., 2000; Yang et al., 2009; Chen et al., 2012). However, UVR8 seems to associate with the promoters of target genes (like HY5) by interacting with histones (Brown et al., 2005), a feature shared by UVR8 and its human homolog RCC1. Nevertheless, there is no evidence thus far to support a direct role of UVR8 in transcriptional regulation. Other important questions that need to be addressed include, but are not limited to, the molecular mechanisms by which COP1 transduces the UV-B signal, and by which UVR8 inhibits COP1-mediated degradation of HY5. Further investigation and ultimate elucidation of these questions and others will undoubtedly shed more light on the signaling mechanisms of the UV-B photomorphogenic pathway.

\section{ACKNOWLEDGMENTS}

We apologize to the authors whose work could not be cited here due to space constraints. This work was supported by grants from the National Basic Research Program of China (973 Program) (Grant No. 2012CB910900), National Institutes of Health of the USA (GM47850), the National Science Foundation (NSF) Plant Genome Program of the USA (DBI0922604), and the Ministry of Agriculture of China (No. 2010ZX08010-003). C.D.N. is a Yale University Brown Postdoctoral Fellow.

\section{ABBREVIATIONS}

COP, constitutively photomorphogenic; FHY, far-red elongated hypocotyl; HY, elongated hypocotyl; RUP, repressor of ultraviolet-B photomorphogenesis; SPA, suppressor of PHYA-105; UV, ultraviolet; UVR, ultraviolet resistance locus

\section{COMPLIANCE WITH ETHICS GUIDELINES}

Jigang Li, Li Yang, Dan Jin, Cynthia D. Nezames, William Terzaghi and Xing Wang Deng declare that they have no conflict of interest.

This article does not contain any studies with human or animal subjects performed by any of the authors.

\section{REFERENCES}

Allen, T., Koustenis, A., Theodorou, G., Somers, D.E., Kay, S.A., Whitelam, G.C., and Devlin, P.F. (2006). Arabidopsis FHY3 specifically gates phytochrome signaling to the circadian clock. Plant Cell 18, 2506-2516.

Brosche, M., Schuler, M.A., Kalbina, I., Connor, L., and Strid, A. (2002). Gene regulation by low level UV-B radiation: identification by DNA array analysis. Photochem Photobiol Sci 1, 656-664.

Brown, B.A., Cloix, C., Jiang, G.H., Kaiserli, E., Herzyk, P., Kliebenstein, D.J., and Jenkins, G.I. (2005). A UV-B-specific signaling component orchestrates plant UV protection. Proc Natl Acad Sci U S A 102, 18225-18230.

Brown, B.A., and Jenkins, G.I. (2008). UV-B signaling pathways with different fluence-rate response profiles are distinguished in mature Arabidopsis leaf tissue by requirement for UVR8, HY5, and HYH. Plant Physiol 146, 576-588.

Casati, P., Stapleton, A.E., Blum, J.E., and Walbot, V. (2006). Genomewide analysis of high-altitude maize and gene knockdown stocks implicates chromatin remodeling proteins in response to UV-B. Plant J 46, 613-627.

Casati, P., and Walbot, V. (2003). Gene expression profiling in response to ultraviolet radiation in maize genotypes with varying flavonoid content. Plant Physiol 132, 1739-1754.

Casati, P., and Walbot, V. (2004). Rapid transcriptome responses of maize (Zea mays) to UV-B in irradiated and shielded tissues. Genome Biol 5, R16.

Chen, F., Shi, X., Chen, L., Dai, M., Zhou, Z., Shen, Y., Li, J., Li, G., Wei, N., and Deng, X.W. (2012). Phosphorylation of FAR-RED ELONGATED HYPOCOTYL1 is a key mechanism defining signaling dynamics of phytochrome $A$ under red and far-red light in Arabidopsis. Plant Cell 24, 1907-1920.

Chen, H., Huang, X., Gusmaroli, G., Terzaghi, W., Lau, O.S., Yanagawa, Y., Zhang, Y., Li, J., Lee, J.H., Zhu, D., et al. (2010). Arabidopsis CULLIN4-damaged DNA binding protein 1 interacts with CONSTITUTIVELY PHOTOMORPHOGENIC1-SUPPRESSOR 
OF PHYA complexes to regulate photomorphogenesis and flowering time. Plant Cell 22, 108-123.

Christie, J.M., Arvai, A.S., Baxter, K.J., Heilmann, M., Pratt, A.J., O'Hara, A., Kelly, S.M., Hothorn, M., Smith, B.O., Hitomi, K., et al. (2012). Plant UVR8 photoreceptor senses UV-B by tryptophan-mediated disruption of cross-dimer salt bridges. Science 335, 1492-1496.

Cloix, C., and Jenkins, G.I. (2008). Interaction of the Arabidopsis UVB-specific signaling component UVR8 with chromatin. Mol Plant 1, 118-128.

Cloix, C., Kaiserli, E., Heilmann, M., Baxter, K.J., Brown, B.A., O'Hara, A., Smith, B.O., Christie, J.M., and Jenkins, G.I. (2012). C-terminal region of the UV-B photoreceptor UVR8 initiates signaling through interaction with the COP1 protein. Proc Natl Acad Sci U S A 109, 16366-16370.

Deng, X.W., Caspar, T., and Quail, P.H. (1991). cop1: a regulatory locus involved in light-controlled development and gene expression in Arabidopsis. Genes Dev 5, 1172-1182.

Deng, X.W., Matsui, M., Wei, N., Wagner, D., Chu, A.M., Feldmann, K.A., and Quail, P.H. (1992). COP1, an Arabidopsis regulatory gene, encodes a protein with both a zinc-binding motif and a $\mathrm{G}$ beta homologous domain. Cell 71, 791-801.

Favory, J.J., Stec, A., Gruber, H., Rizzini, L., Oravecz, A., Funk, M., Albert, A., Cloix, C., Jenkins, G.I., Oakeley, E.J., et al. (2009). Interaction of COP1 and UVR8 regulates UV-B-induced photomorphogenesis and stress acclimation in Arabidopsis. EMBO J 28, 591-601.

Feher, B., Kozma-Bognar, L., Kevei, E., Hajdu, A., Binkert, M., Davis, S.J., Schafer, E., Ulm, R., and Nagy, F. (2011). Functional interaction of the circadian clock and UV RESISTANCE LOCUS 8-controlled UV-B signaling pathways in Arabidopsis thaliana. Plant $\mathrm{J} 67$, $37-48$.

Genoud, T., Schweizer, F., Tscheuschler, A., Debrieux, D., Casal, J.J., Schafer, E., Hiltbrunner, A., and Fankhauser, C. (2008). FHY1 mediates nuclear import of the light-activated phytochrome A photoreceptor. PLoS Genet 4, e1000143.

Gonzalez Besteiro, M.A., Bartels, S., Albert, A., and Ulm, R. (2011). Arabidopsis MAP kinase phosphatase 1 and its target MAP kinases 3 and 6 antagonistically determine UV-B stress tolerance, independent of the UVR8 photoreceptor pathway. Plant J 68, 727-737.

Gruber, H., Heijde, M., Heller, W., Albert, A., Seidlitz, H.K., and Ulm, R. (2010). Negative feedback regulation of UV-B-induced photomorphogenesis and stress acclimation in Arabidopsis. Proc Natl Acad Sci U S A 107, 20132-20137.

Heijde, M., and Ulm, R. (2012). UV-B photoreceptor-mediated signalling in plants. Trends Plant Sci 17, 230-237.

Heijde, M., and Ulm, R. (2013). Reversion of the Arabidopsis UV-B photoreceptor UVR8 to the homodimeric ground state. Proc Natl Acad Sci U S A 110, 1113-1118.

Heilmann, M., and Jenkins, G.I. (2013). Rapid reversion from monomer to dimer regenerates the ultraviolet-B photoreceptor UV RESISTANCE LOCUS8 in intact Arabidopsis plants. Plant Physiol 161, 547-555.

Hiltbrunner, A., Tscheuschler, A., Viczian, A., Kunkel, T., Kircher, S., and Schafer, E. (2006). FHY1 and FHL act together to mediate nuclear accumulation of the phytochrome A photoreceptor. Plant Cell Physiol 47, 1023-1034.

Hiltbrunner, A., Viczian, A., Bury, E., Tscheuschler, A., Kircher, S., Toth, R., Honsberger, A., Nagy, F., Fankhauser, C., and Schafer, E.
(2005). Nuclear accumulation of the phytochrome A photoreceptor requires FHY1. Curr Biol 15, 2125-2130.

Hoecker, U., and Quail, P.H. (2001). The phytochrome A-specific signaling intermediate SPA1 interacts directly with COP1, a constitutive repressor of light signaling in Arabidopsis. J Biol Chem 276, 38173-38178.

Hoecker, U., Xu, Y., and Quail, P.H. (1998). SPA1: a new genetic locus involved in phytochrome A-specific signal transduction. Plant Cell 10, 19-33.

Holm, M., Ma, L.G., Qu, L.J., and Deng, X.W. (2002). Two interacting bZIP proteins are direct targets of COP1-mediated control of light-dependent gene expression in Arabidopsis. Genes Dev 16, 1247-1259.

Huang, X., Ouyang, X., Yang, P., Lau, O.S., Li, G., Li, J., Chen, H., and Deng, X.W. (2012). Arabidopsis FHY3 and HY5 positively mediate induction of COP1 transcription in response to photomorphogenic UV-B light. Plant Cell 24, 4590-4606.

Hudson, M., Ringli, C., Boylan, M.T., and Quail, P.H. (1999). The FAR1 locus encodes a novel nuclear protein specific to phytochrome $A$ signaling. Genes Dev 13, 2017-2027.

Jang, I.C., Henriques, R., Seo, H.S., Nagatani, A., and Chua, N.H. (2010). Arabidopsis PHYTOCHROME INTERACTING FACTOR proteins promote phytochrome $B$ polyubiquitination by COP1 E3 ligase in the nucleus. Plant Cell 22, 2370-2383.

Jenkins, G.I. (2009). Signal transduction in responses to UV-B radiation. Annu Rev Plant Biol 60, 407-431.

Jiang, L., Wang, Y., Bjorn, L.O., and Li, S. (2009). Arabidopsis RADICAL-INDUCED CELL DEATH1 is involved in UV-B signaling. Photochem Photobiol Sci 8, 838-846.

Jiang, L., Wang, Y., Li, Q.F., Bjorn, L.O., H, J.X., and Li, S. (2012). Arabidopsis STO/BBX24 negatively regulates UV-B signaling by interacting with COP1 and repressing HY5 transcriptional activity. Cell Res 22, 1046-1057.

Kaiserli, E., and Jenkins, G.I. (2007). UV-B promotes rapid nuclear translocation of the Arabidopsis UV-B specific signaling component UVR8 and activates its function in the nucleus. Plant Cell 19, 2662-2673.

Kilian, J., Whitehead, D., Horak, J., Wanke, D., Weinl, S., Batistic, O., D'Angelo, C., Bornberg-Bauer, E., Kudla, J., and Harter, K. (2007). The AtGenExpress global stress expression data set: protocols, evaluation and model data analysis of UV-B light, drought and cold stress responses. Plant J 50, 347-363.

Kim B.C., Tennessen D.J., Last R.L. (1998). UV-B-induced photomorphogenesis in Arabidopsis thaliana. Plant $\mathrm{J} \mathrm{15,667-674.}$

Kliebenstein, D.J., Lim, J.E., Landry, L.G., and Last, R.L. (2002). Arabidopsis UVR8 regulates ultraviolet-B signal transduction and tolerance and contains sequence similarity to human regulator of chromatin condensation 1. Plant Physiol 130, 234-243.

Koornneef, M., Rolff, E., and Spruit, C.J.P. (1980). Genetic control of light-inhibited hypocotyl elongation in Arabidopsis thaliana (L.) Heynh Z Pflanzenphysiol 100, 147-160.

Lau, O.S., and Deng, X.W. (2012). The photomorphogenic repressors COP1 and DET1: 20 years later. Trends Plant Sci 17, 584-593.

Laubinger, S., Fittinghoff, K., and Hoecker, U. (2004). The SPA quartet: a family of WD-repeat proteins with a central role in suppression of photomorphogenesis in Arabidopsis. Plant Cell 16, 2293-2306.

Laubinger, S., and Hoecker, U. (2003). The SPA1-like proteins SPA3 
and SPA4 repress photomorphogenesis in the light. Plant $\mathrm{J} 35$, 373-385.

Lee, J., He, K., Stolc, V., Lee, H., Figueroa, P., Gao, Y., Tongprasit, W., Zhao, H., Lee, I., and Deng, X.W. (2007). Analysis of transcription factor HY5 genomic binding sites revealed its hierarchical role in light regulation of development. Plant Cell 19, 731-749.

Lee, J.H., Terzaghi, W., Gusmaroli, G., Charron, J.B., Yoon, H.J., Chen, H., He, Y.J., Xiong, Y., and Deng, X.W. (2008). Characterization of Arabidopsis and rice DWD proteins and their roles as substrate receptors for CUL4-RING E3 ubiquitin ligases. Plant Cell 20, 152-167.

Li, G., Siddiqui, H., Teng, Y., Lin, R., Wan, X.Y., Li, J., Lau, O.S., Ouyang, X., Dai, M., Wan, J., et al. (2011). Coordinated transcriptional regulation underlying the circadian clock in Arabidopsis. Nat Cell Biol 13, 616-622.

Li, J., Li, G., Gao, S., Martinez, C., He, G., Zhou, Z., Huang, X., Lee, J.H., Zhang, H., Shen, Y., et al. (2010). Arabidopsis transcription factor ELONGATED HYPOCOTYL5 plays a role in the feedback regulation of phytochrome A signaling. Plant Cell 22, 3634-3649.

Lin, R., Ding, L., Casola, C., Ripoll, D.R., Feschotte, C., and Wang, H. (2007). Transposase-derived transcription factors regulate light signaling in Arabidopsis. Science 318, 1302-1305.

Ma, L., Gao, Y., Qu, L., Chen, Z., Li, J., Zhao, H., and Deng, X.W. (2002). Genomic evidence for COP1 as a repressor of light-regulated gene expression and development in Arabidopsis. Plant Cell 14, 23832398.

Martinez-Garcia, J.F., Huq, E., and Quail, P.H. (2000). Direct targeting of light signals to a promoter element-bound transcription factor. Science 288, 859-863.

Morales L.O., Brosché M., Vainonen J., Jenkins G.I., Wargent J.J., Sipari N., Strid A., Lindfors A.V., Tegelberg R., Aphalo P.J. (2013). Multiple roles for UV RESISTANCE LOCUS8 in regulating gene expression and metabolite accumulation in Arabidopsis under solar ultraviolet radiation. Plant Physiol 161, 744-759.

O'Hara, A., and Jenkins, G.I. (2012). In vivo function of tryptophans in the Arabidopsis UV-B photoreceptor UVR8. Plant Cell 24, 37553766.

Oravecz, A., Baumann, A., Mate, Z., Brzezinska, A., Molinier, J., Oakeley, E.J., Adam, E., Schafer, E., Nagy, F., and Ulm, R. (2006). CONSTITUTIVELY PHOTOMORPHOGENIC1 is required for the UV-B response in Arabidopsis. Plant Cell 18, 1975-1990.

Osterlund, M.T., Hardtke, C.S., Wei, N., and Deng, X.W. (2000). Targeted destabilization of HY5 during light-regulated development of Arabidopsis. Nature 405, 462-466.

Ouyang, X., Li, J., Li, G., Li, B., Chen, B., Shen, H., Huang, X., Mo, X., Wan, X., Lin, R., et al. (2011). Genome-wide binding site analysis of FAR-RED ELONGATED HYPOCOTYL3 reveals its novel function in Arabidopsis development. Plant Cell 23, 2514-2535.

Oyama, T., Shimura, Y., and Okada, K. (1997). The Arabidopsis HY5 gene encodes a bZIP protein that regulates stimulus-induced development of root and hypocotyl. Genes Dev 11, 2983-2995.

Rizzini, L., Favory, J.J., Cloix, C., Faggionato, D., O'Hara, A., Kaiserli, E., Baumeister, R., Schafer, E., Nagy, F., Jenkins, G.I., et al. (2011). Perception of UV-B by the Arabidopsis UVR8 protein. Science 332, 103-106.

Saijo, Y., Sullivan, J.A., Wang, H., Yang, J., Shen, Y., Rubio, V., Ma, L., Hoecker, U., and Deng, X.W. (2003). The COP1-SPA1 interaction defines a critical step in phytochrome A-mediated regulation of HY5 activity. Genes Dev 17, 2642-2647.

Saijo, Y., Zhu, D., Li, J., Rubio, V., Zhou, Z., Shen, Y., Hoecker, U., Wang, H., and Deng, X.W. (2008). Arabidopsis COP1/SPA1 complex and FHY1/FHY3 associate with distinct phosphorylated forms of phytochrome $A$ in balancing light signaling. Mol Cell 31, 607-613.

Seo, H.S., Watanabe, E., Tokutomi, S., Nagatani, A., and Chua, N.H. (2004). Photoreceptor ubiquitination by COP1 E3 ligase desensitizes phytochrome A signaling. Genes Dev 18, 617-622.

Seo, H.S., Yang, J.Y., Ishikawa, M., Bolle, C., Ballesteros, M.L., and Chua, N.H. (2003). LAF1 ubiquitination by COP1 controls photomorphogenesis and is stimulated by SPA1. Nature 423, 995-999.

Shalitin, D., Yang, H., Mockler, T.C., Maymon, M., Guo, H., Whitelam, G.C., and Lin, C. (2002). Regulation of Arabidopsis cryptochrome 2 by blue-light-dependent phosphorylation. Nature 417, 763-767.

Stirnberg, P., Zhao, S., Williamson, L., Ward, S., and Leyser, O. (2012). FHY3 promotes shoot branching and stress tolerance in Arabidopsis in an AXR1-dependent manner. Plant J 71, 907-920.

Subramanian, C., Kim, B.H., Lyssenko, N.N., Xu, X., Johnson, C.H., and von Arnim, A.G. (2004). The Arabidopsis repressor of light signaling, COP1, is regulated by nuclear exclusion: mutational analysis by bioluminescence resonance energy transfer. Proc Natl Acad Sci U S A 101, 6798-6802.

Tang, W., Wang, W., Chen, D., Ji, Q., Jing, Y., Wang, H., and Lin, R. (2012). Transposase-derived proteins FHY3/FAR1 interact with PHYTOCHROME-INTERACTING FACTOR1 to regulate chlorophyll biosynthesis by modulating HEMB1 during deetiolation in Arabidopsis. Plant Cell 24, 1984-2000.

Ulm, R., Baumann, A., Oravecz, A., Mate, Z., Adam, E., Oakeley, E.J., Schafer, E., and Nagy, F. (2004). Genome-wide analysis of gene expression reveals function of the bZIP transcription factor HY5 in the UV-B response of Arabidopsis. Proc Natl Acad Sci U S A 101, 1397-1402.

Ulm, R., and Nagy, F. (2005). Signalling and gene regulation in response to ultraviolet light. Curr Opin Plant Biol 8, 477-482.

van Nocker, S., and Ludwig, P. (2003). The WD-repeat protein superfamily in Arabidopsis: conservation and divergence in structure and function. BMC Genomics 4, 50.

von Arnim, A.G., and Deng, X.W. (1994). Light inactivation of Arabidopsis photomorphogenic repressor COP1 involves a cell-specific regulation of its nucleocytoplasmic partitioning. Cell 79, 1035-1045.

Wang, H., and Deng, X.W. (2002). Arabidopsis FHY3 defines a key phytochrome A signaling component directly interacting with its homologous partner FAR1. EMBO J 21, 1339-1349.

Whitelam, G.C., Johnson, E., Peng, J., Carol, P., Anderson, M.L., Cowl, J.S., and Harberd, N.P. (1993). Phytochrome A null mutants of Arabidopsis display a wild-type phenotype in white light. Plant Cell 5, 757-768.

Wu, D., Hu, Q., Yan, Z., Chen, W., Yan, C., Huang, X., Zhang, J., Yang, P., Deng, H., Wang, J., et al. (2012). Structural basis of ultraviolet-B perception by UVR8. Nature 484, 214-219.

Yang, S.W., Jang, I.C., Henriques, R., and Chua, N.H. (2009). FARRED ELONGATED HYPOCOTYL1 and FHY1-LIKE associate with the Arabidopsis transcription factors LAF1 and HFR1 to transmit phytochrome A signals for inhibition of hypocotyl elongation. Plant Cell 21, 1341-1359.

Yi, C., and Deng, X.W. (2005). COP1 - from plant photomorphogen- 
esis to mammalian tumorigenesis. Trends Cell Biol 15, 618-625.

Zhang, H., He, H., Wang, X., Wang, X., Yang, X., Li, L., and Deng, X.W. (2011). Genome-wide mapping of the HY5-mediated gene networks in Arabidopsis that involve both transcriptional and posttranscriptional regulation. Plant J 65, 346-358.
Zhu, D., Maier, A., Lee, J.H., Laubinger, S., Saijo, Y., Wang, H., Qu, L.J., Hoecker, U., and Deng, X.W. (2008). Biochemical characterization of Arabidopsis complexes containing CONSTITUTIVELY PHOTOMORPHOGENIC1 and SUPPRESSOR OF PHYA proteins in light control of plant development. Plant Cell 20, 2307-2323. 